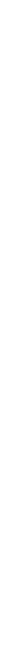

\title{
Digital Acrylics
}

$\begin{aligned} \text { Authors: } & \text { Manfred Kranz } \\ \text { Submitted: } & \text { 22. June } 2015 \\ \text { Published: } & \text { 23. June } 2015 \\ \text { Volume: } & 2 \\ \text { Issue: } & 4 \\ \text { Keywords: } & \text { fotorealistische Illustration, Digital Acrylics } \\ \text { DOI: } & 10.17160 \text { /josha.2.4.43 }\end{aligned}$

\section{JOSHA Humanities and Arts}




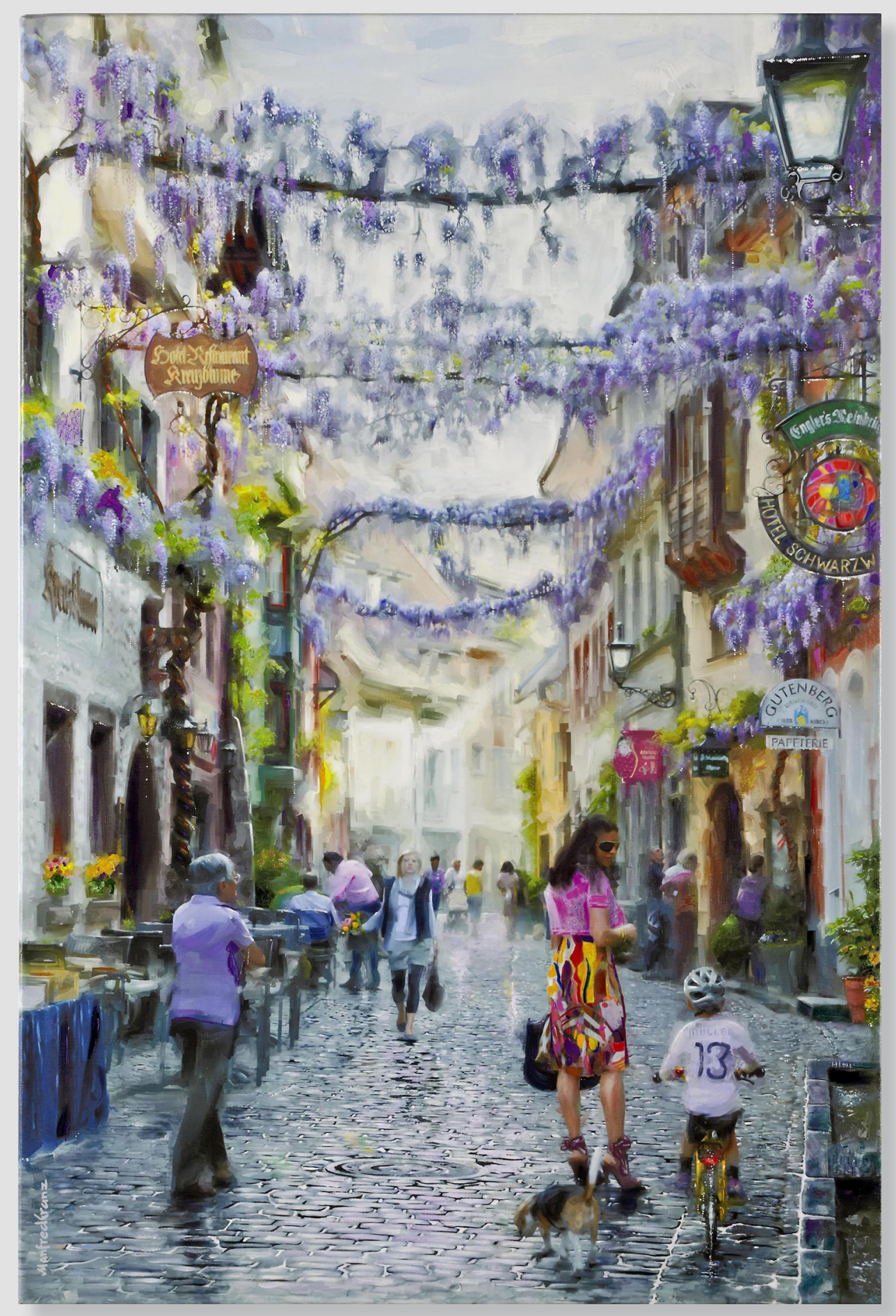

$\left\{\begin{array}{l}\text { Manfred Kranz AGD } \\ \text { March-Hugstetten (Germany) } \\ \text { www.kranz-illustration.com } \\ \text { info@kranz-illustration.com }\end{array}\right.$

Glyzinienblüte in der Konviktstraße

\section{Vita}

Geboren 1950 in Freiburg, verbrachte ich meine Kindheit und Schulzeit ab 1956 in Hugstetten bei Freiburg i. Br. Meine Schriftsetzerlehre führte mit anschließendem Grafikdesign-Studium schließlich zu meinem Beruf.

Nach einigen Berufsjahren mit unumgänglich autodidakter Weiterbildung in den Sparten technische/figürliche Illustration und Fotografie erfolgte meine Spezialisierung auf Darstellung fotorealistisch gezeichneter Bilder mit klassischen Mitteln und Werkzeugen.

Einsatzgebiete: nicht oder nur schwierig zu fotografierende Motive/Szenen.

\section{Nische gefunden}

Anfang der Achtzigerjahre beim Spezialgebiet fotorealistische Illustration gelandet. Schwerpunkte: Technik, Medizin, Forschung, Architektur etc. Das zeigen zunächst die 18 kleinen Bildchen und die 3 Portraitzeichnungen auf Seite 2. Es sind fotorealistisch gezeichnete Auftragsarbeiten aus meinem Berufsalltag (Seite 2). Diese gehören daher nicht zu meiner Bilderserie „Digital Acrylics". Aber sie leiten von meiner beruflichen Tätigkeit über zu den „Digital Acrylics" der Folgeseiten. 16 dieser 21 Bilder übrigens sind "handgemacht" mit klassischem Werkzeug auf Zeichenkarton. 


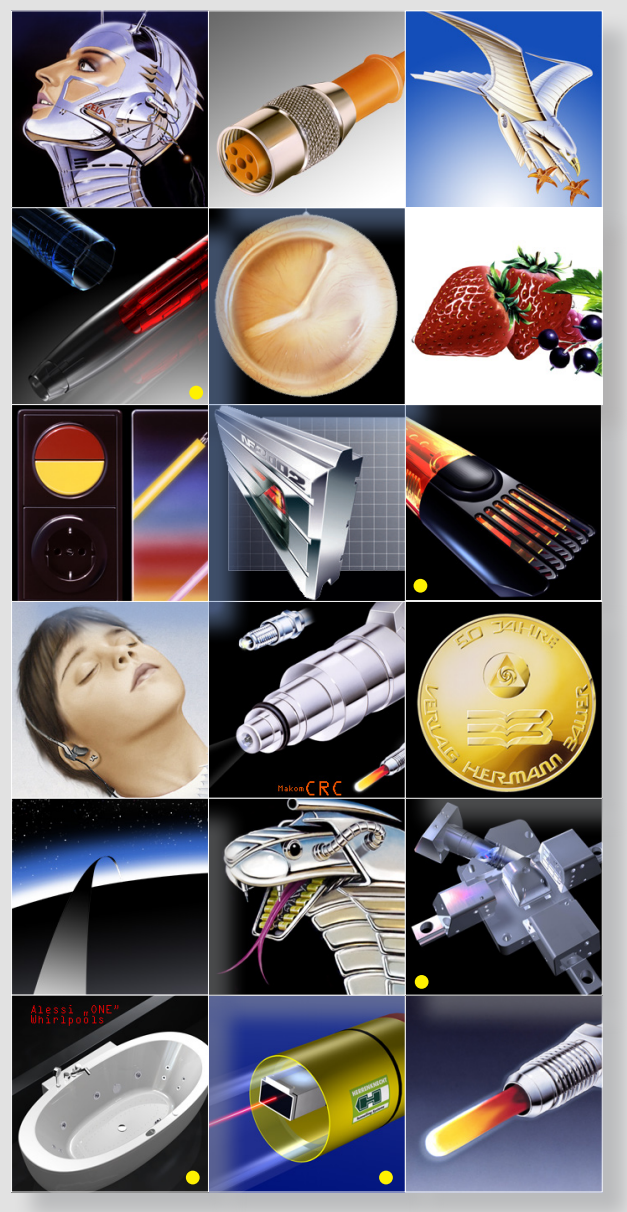

Das gelbe Pünktchen auf 5 Bildern bedeutet, dass die jeweilige Zeichnung nicht mit klassischem Werkzeug, sondern mit einem Malprogramm auf dem Computer erstellt wurde. Ein entsprechendes Malprogramm war dazu ab der 2. Hälfte der Neunzigerjahre so weit ausgereift und verfügbar.

Nach wie vor entsteht ein computergeneriertes Bild nicht von selbst. Sein Entstehungsprozess beginnt noch immer im Kopf. Seine Ausführung geschieht mit digitalen Werkzeugen.

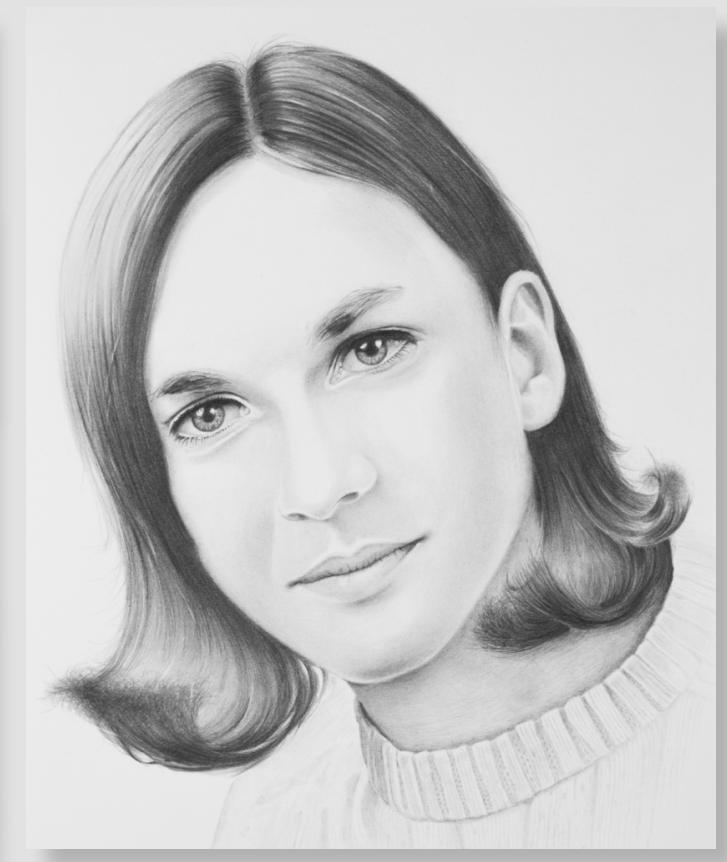

Kleine Bilder links drüben sowie die 3 Portraitzeichnungen:

Illustrationen als Auftragsarbeiten aus meinem Berufsalltag. Mehrheitlich ausgeführt mit

klassischen Zeichenmaterialien

(flüssige und pastose Acrylfarbe, Bleistifte, Buntstifte, Pinsel, Ziehfeder, Kreiden, Radierer,

Airbrush) auf Reinzeichenkar-

ton resp. speziellen Zeichenpapiersorten.

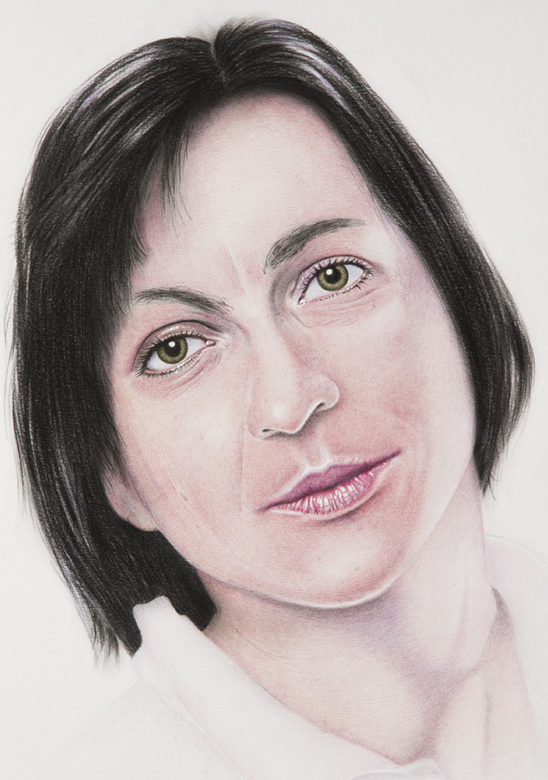

Buntstift

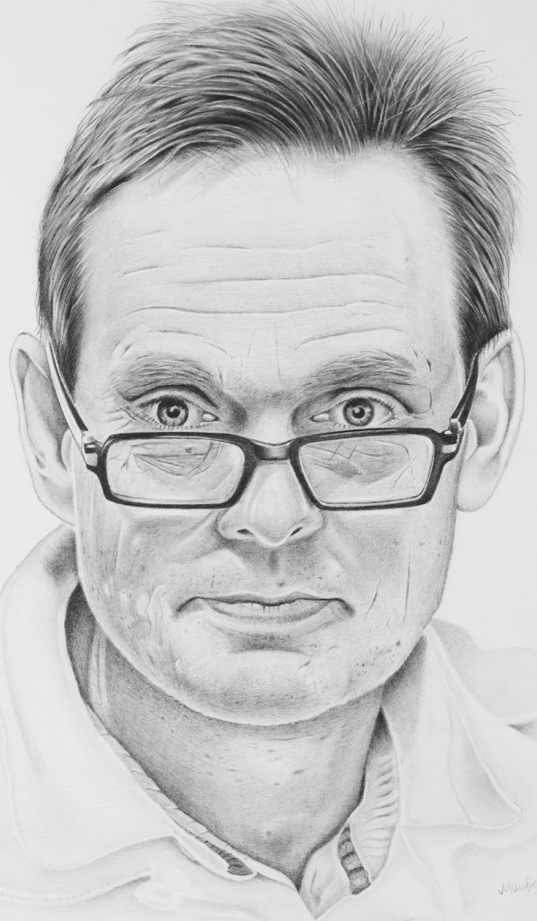

Bleistift
Zwischendurch sind auch mal Portraitzeichnungen gefragt: Portraits mit Bleistift, Pinsel, Buntstift, Zirkel - ganz ohne Computer

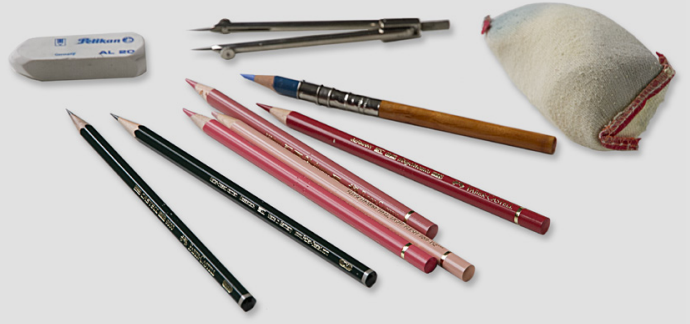




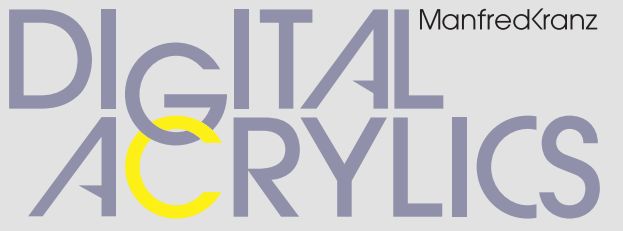

\section{Zeit für Neues}

Nach inzwischen 47 Berufsjahren nehme ich mir neben meinen Auftragsarbeiten etwas mehr Zeit für freie Arbeiten: Betont impressiv gestaltete ästhetische Bilder - basierend auch auf den erlernten Techniken meines bisherigen Berufslebens. Seiten 3 bis 8 zeigen einige dieser seit 2010 entstandenen großformatigen "Digital Acrylics“. Das ist als Mix aus digitaler Fotografie, der Anwendung eines digitalen Malprogramms und akzentuierender klassischer Malerei zu verstehen. Dieser Medien-Mix ist zunächst Grundlage meiner ersten freien Bilderserie.

\section{Warum Acryl?}

Erstklassige Acrylfarbe besitzt einzigartige Eigenschaften. Ich schätze sie seit Jahrzehnten. Jetzt aber ganz besonders im Hinblick auf meine künftigen freien Arbeiten wegen Ihrer Robustheit und Alterungsbeständigkeit etwa im Vergleich zu Ölfarben.

\section{Ausblick}

Meine nächste Serie entsteht mit Spachtel und Pinsel auf Leinwand genauer gesagt, auf schwerem Baumwolltuch. Es ist zwar unüblich, halbfertige Werke zu präsentieren - aber in diesem Fall habe ich mich dennoch dazu entschlossen. Das Herbstmotiv „Burg Landeck“ bei Teningen (Süd-

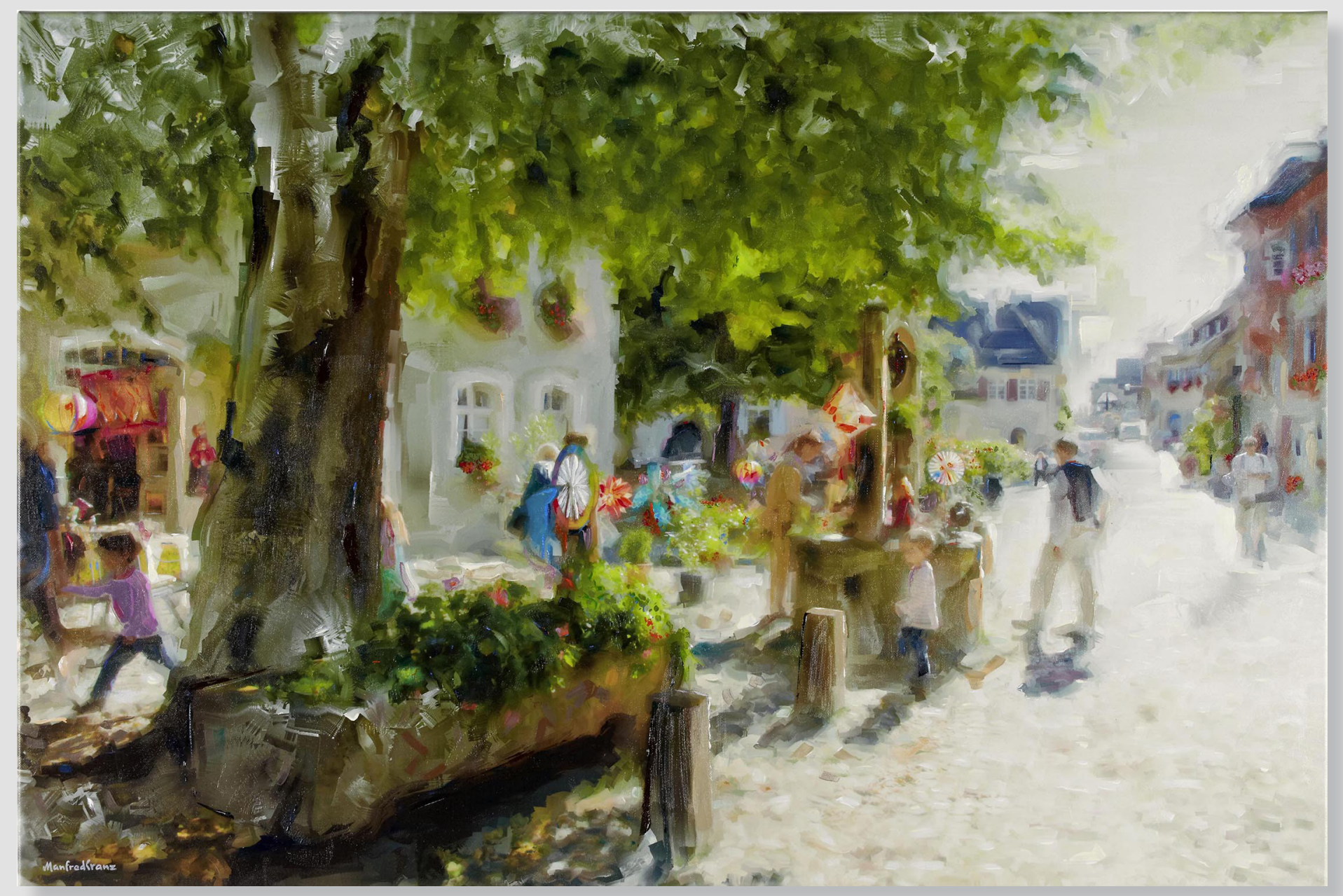

Marionettenladen Burkheim, Mittelstadt Spätsommer 2010 Fine-Art-Print-Leinwand auf Keilrahmen $120 \times 80 \mathrm{~cm}$

baden) auf Seite 8 verschafft einen ersten,

wenn auch unvollständigen Eindruck von der Anwendung dieser Mischtechnik.

Manfred Kranz

March, im Mai 2015 


\section{DIG RYLICS}

Panorama Burkheim

März 2011

Keilrahmen, $120 \times 60 \mathrm{~cm}$

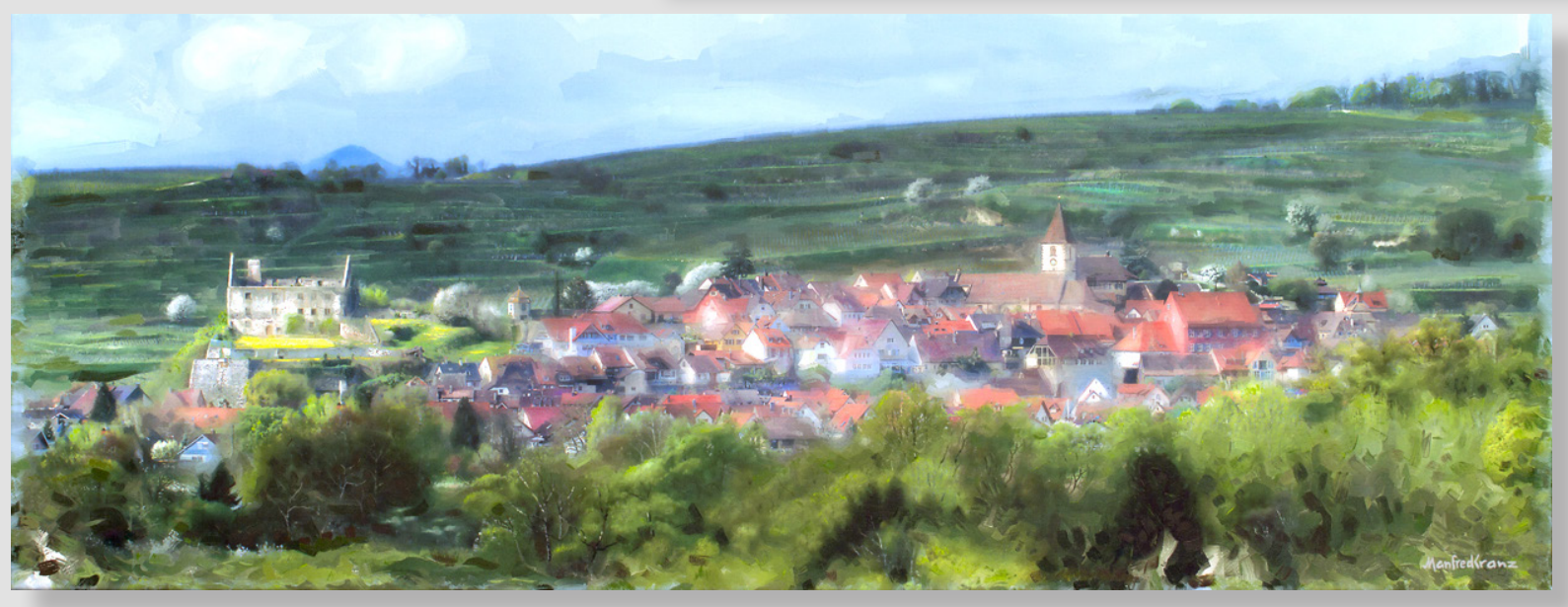

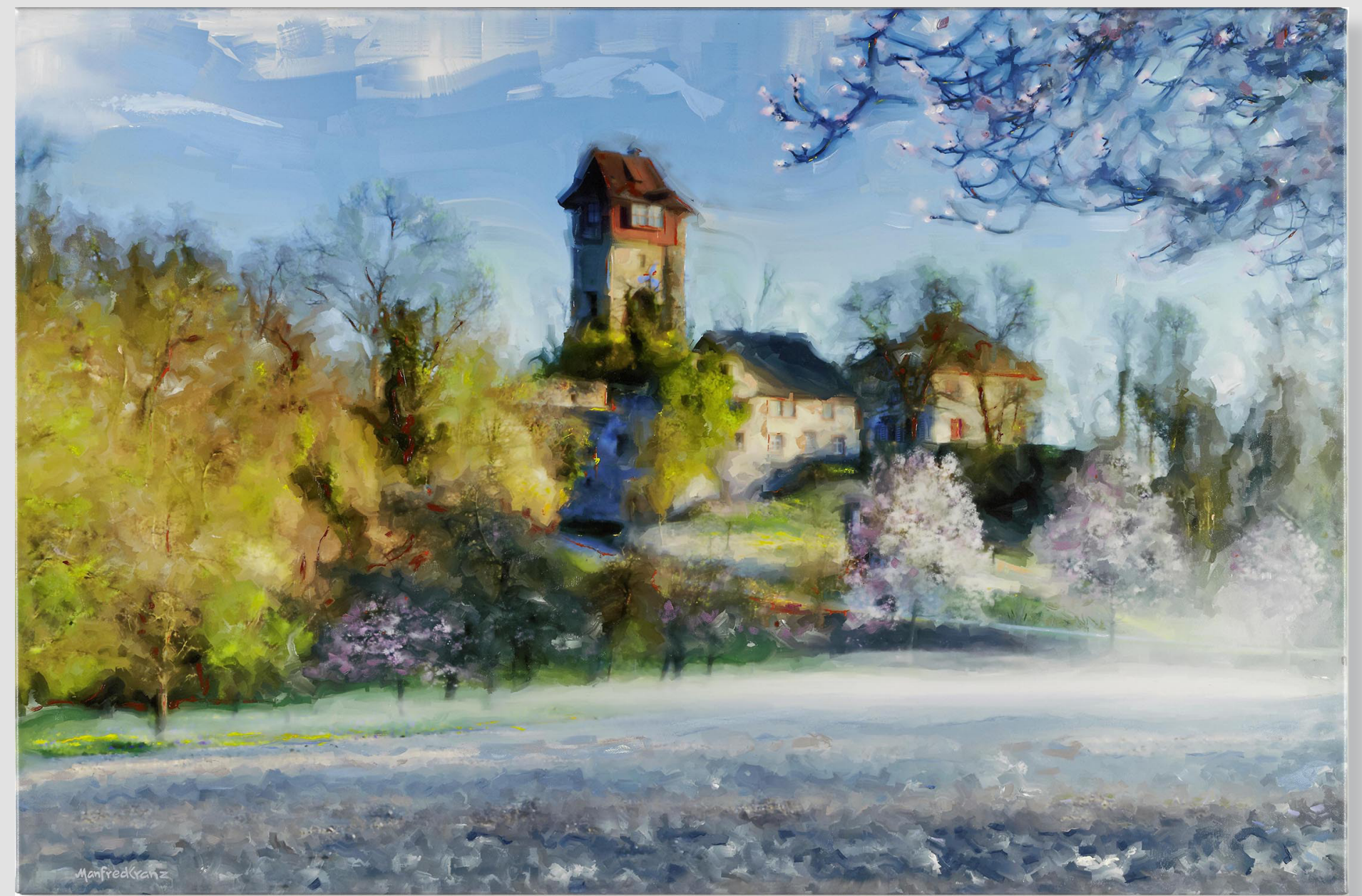

Burgruine Sponeck Jechtingen

März 2011

Fine-Art-Print-Leinwand

auf Keilrahmen $120 \times 80 \mathrm{~cm}$ 


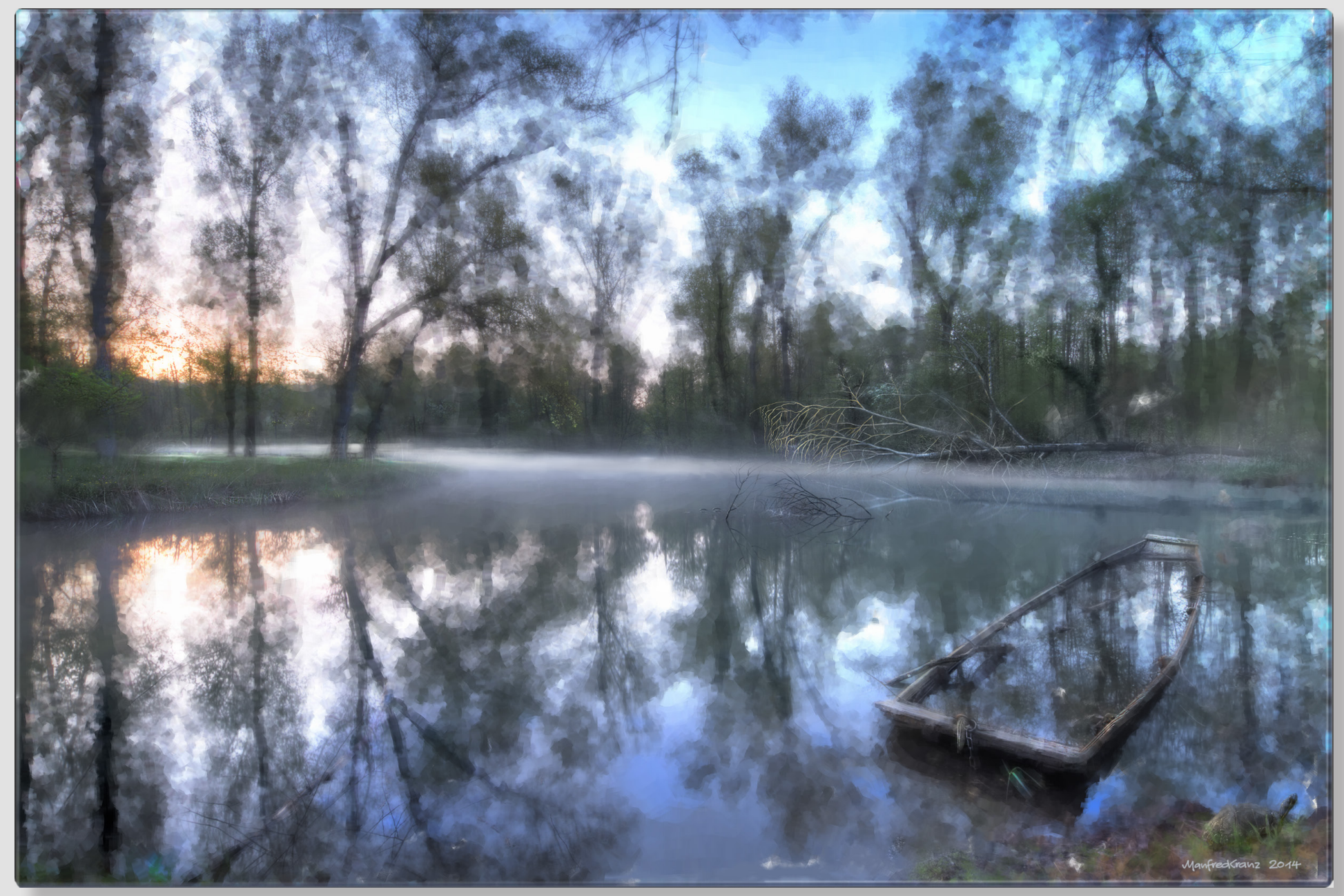

Altrhein bei Burkheim April 2012

Fine-Art-Print-Leinwand

auf Keilrahmen $120 \times 80 \mathrm{~cm}$ 


\section{DIG RÝLICS}

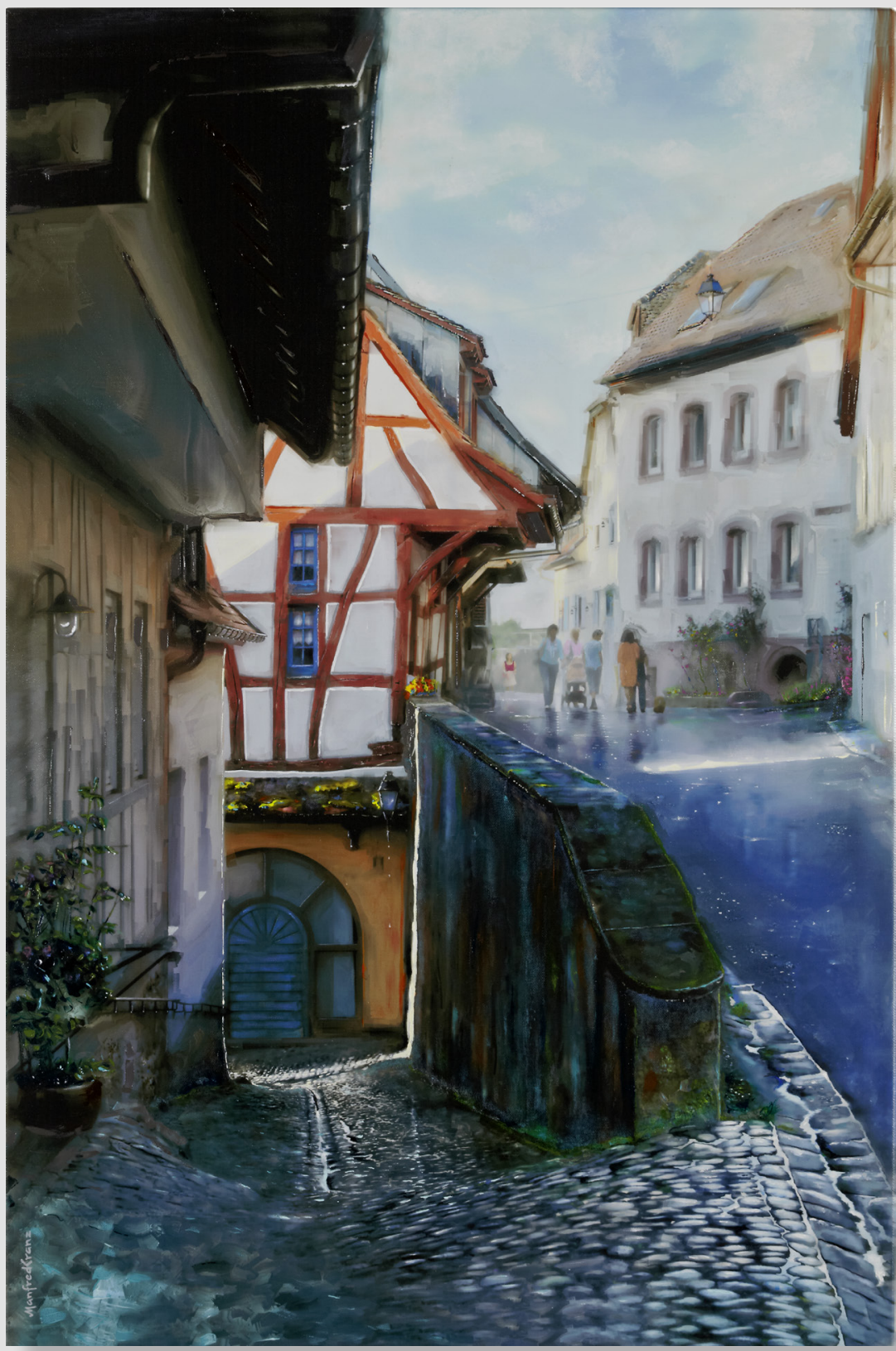

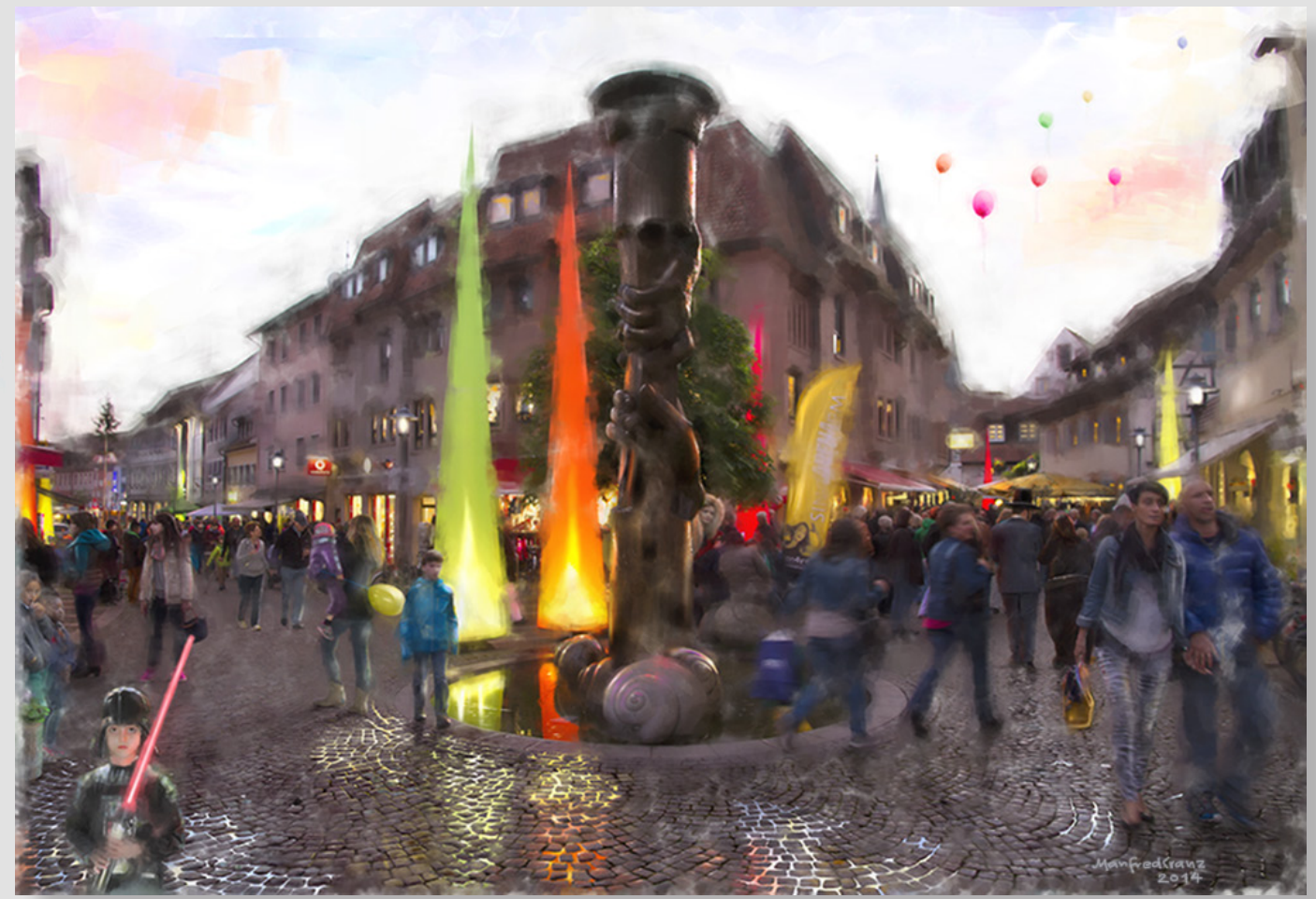

Emmendinger Einkaufsnacht 2014 Emmendingen

Mai 2014

Fine-Art-Print-Leinwand auf Keilrahmen $120 \times 80 \mathrm{~cm}$ 

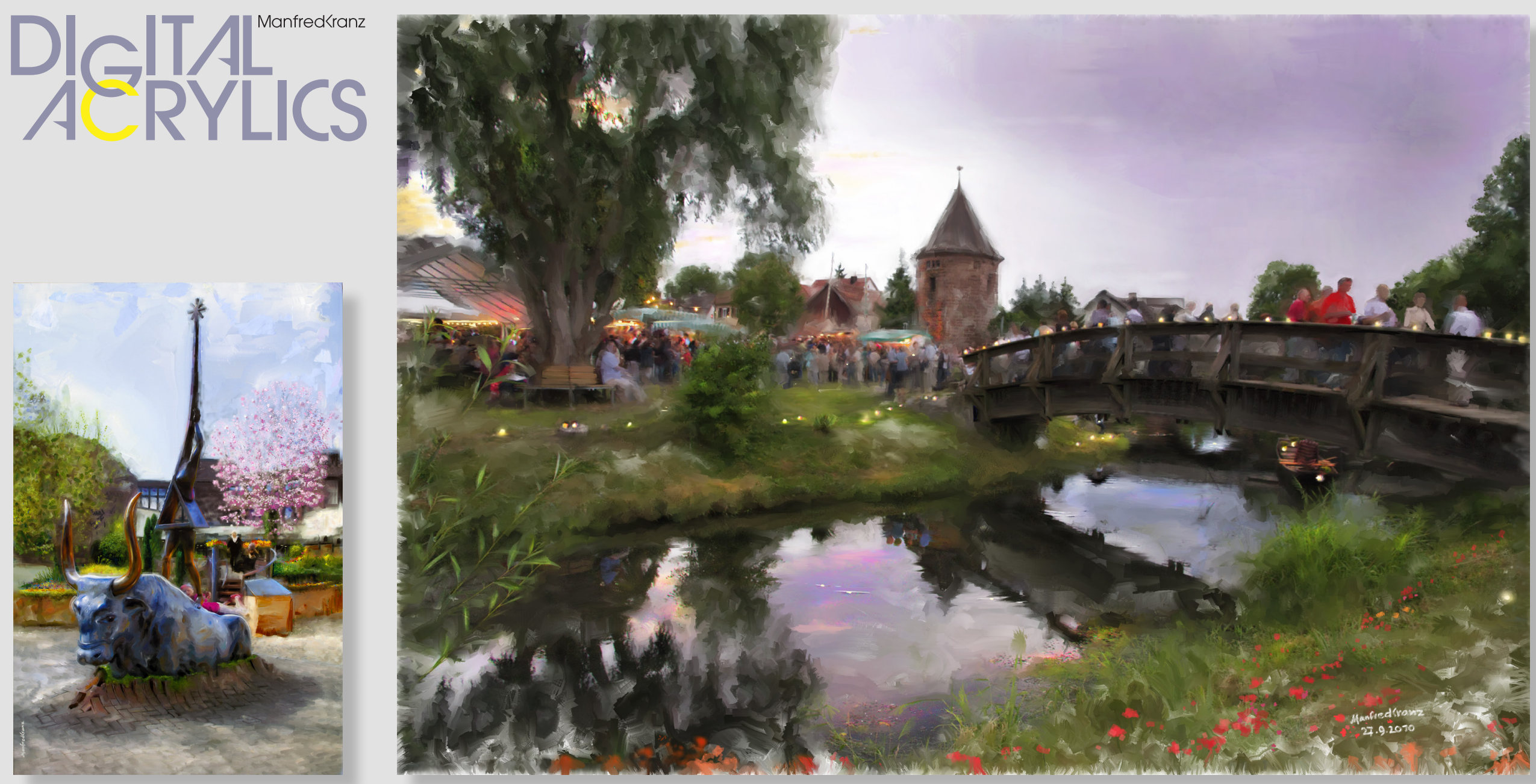

Europastier

Münsterplatz, Breisach

Bildhauer: Helmut Lutz

April 2012

Fine-Art-Print-Leinwand

auf Keilrahmen $80 \times 120 \mathrm{~cm}$

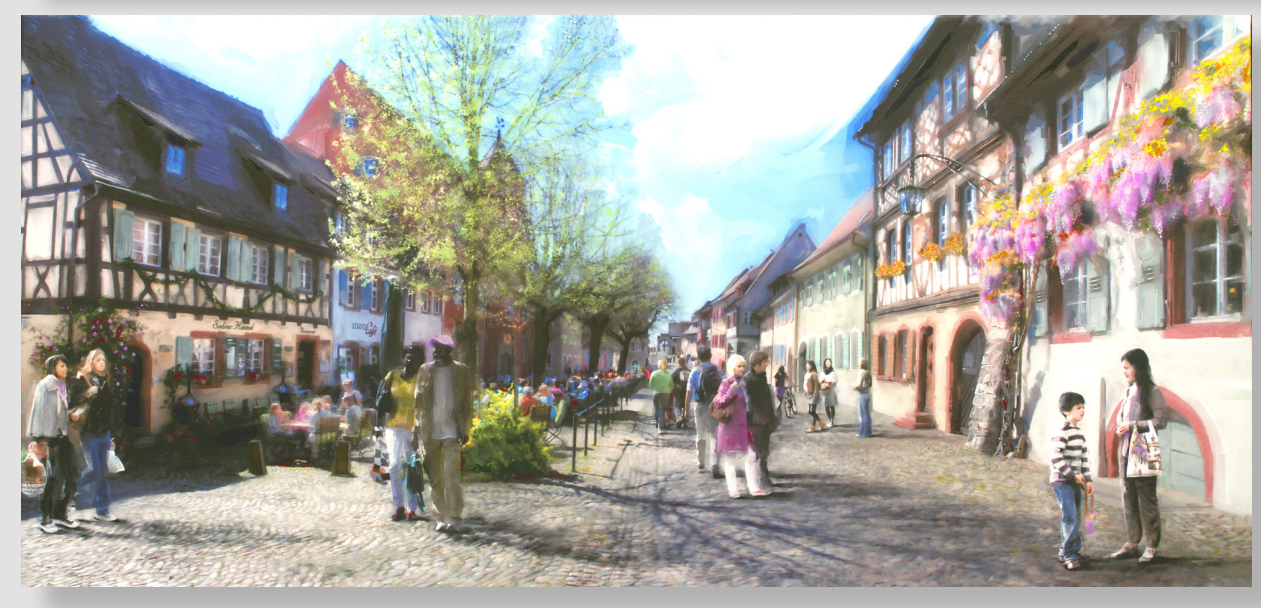

Eichstetter ${ }_{n}$ Wein-Nacht

Eichstetten am Kaiserstuh Juli 2010

Fine-Art-Print-Leinwand

auf Keilrahmen $60 \times 40 \mathrm{~cm}$

Burkheimer Mittelstadt April 2012

Fine-Art-Print-Leinwand

auf Keilrahmen $120 \times 60 \mathrm{~cm}$ 


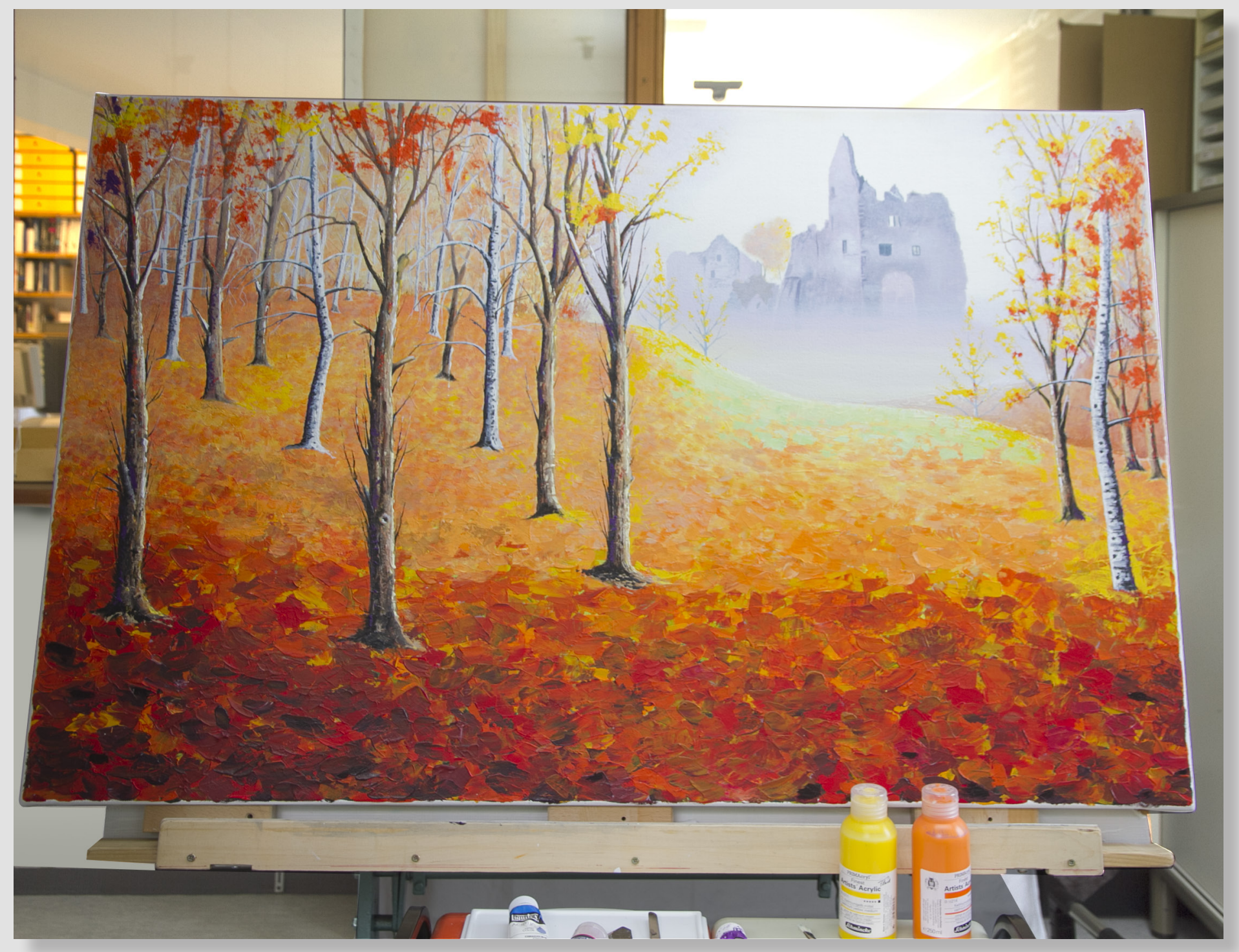

Noch auf der Staffelei:

Zwischenstadium eines noch unvollendeten Motivs

Burgruine Landeck bei Teningen (Südbaden)

Herbst 2014

Spachtel / Pinsel auf Baumwolltuch

Keilrahmen $120 \times 80 \mathrm{~cm}$

Manfred Kranz AGD

March-Hugstetten (Germany)

www.kranz-illustration.com

info@kranz-illustration.com 